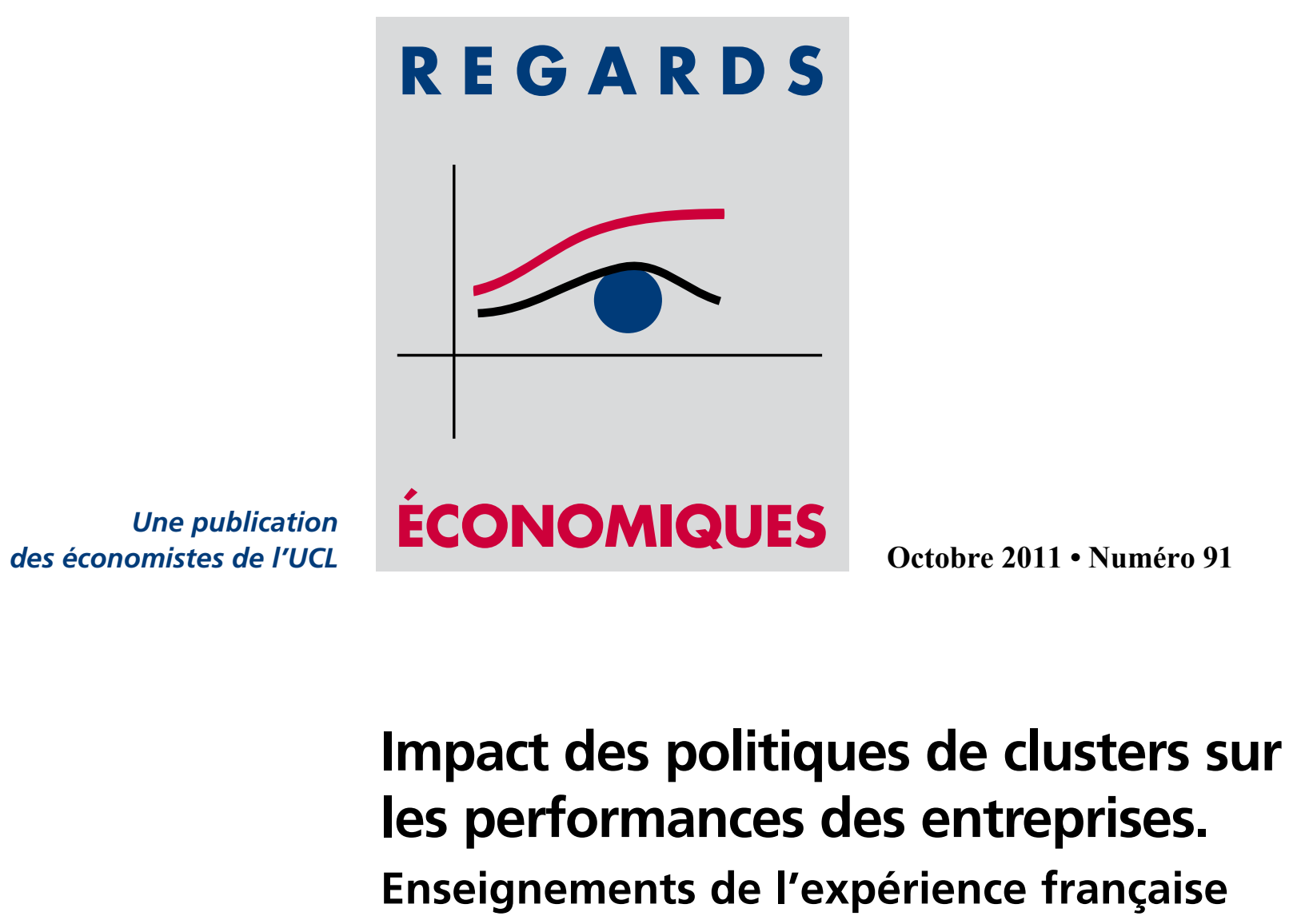

Les politiques de clusters sont aujourd'hui présentées comme l'instrument incontournable du développement local et de la compétitivité des Etats.

Pourtant, leur analyse révèle une très grande diversité qui laisse parfois douter de leur cohérence. L'évaluation des Systèmes Productifs Locaux et des Pôles de compétitivité français illustre bien cette hétérogénéité. Le corollaire de ceci est qu'il est difficile d'avoir un discours normatif sur les politiques de clusters. Les politiques de clusters ne sont pas bonnes ou mauvaises en soi. Il y a en revanche des initiatives qui marchent et d'autres pas. L'évaluation permet d'identifier les succès et les échecs, mais fournit aussi un éclairage sur le dispositif lui-même de la politique, utile à son évolution et à son amélioration.

\section{Florian Mayneris}

D'après Michael Porter (2000), «un cluster est un regroupement d'entreprises et d'institutions associées géographiquement proches, opérant dans un champ d'activité particulier et reliées par des points communs et des complémentarités». L'existence de telles agglomérations productives n'est pas un phénomène nouveau pour les économistes. Dès la fin du XIXème siècle, Alfred Marshall s'étonnait, dans ses Principes d'économie politique, de la concentration en Angleterre des industries de la bonneterie et de la coutellerie dans les régions de Northampton et de Sheffield, concentration qu'aucune dépendance vis-à-vis de matières premières spatialement localisées ne permettait d'expliquer. Ce sont les externalités positives qui s'exercent entre des entreprises géographiquement proches qui permettent de rendre compte, selon Marshall, de ces «districts industriels» : bassin de main-d'œuvre spécialisée, partage d'infrastructures ou d'inputs spécifiques, transferts de connaissances.

L'utilisation du concept de cluster ou de district industriel par les pouvoirs publics est en revanche beaucoup plus récente, puisqu'elle remonte aux années 1980 seulement. Alors que l'industrie commence à décliner dans la plupart des pays développés sous les effets conjugués des chocs pétroliers et de la concurrence de plus en plus forte des pays émergents, un pays semble bien résister, l'Italie, notamment dans des secteurs réputés plutôt traditionnels tels que l'habillement, la chaussure ou encore la fabrication de meubles. Or la localisation des entreprises industrielles italiennes obéit, encore aujourd'hui, à une forte logique de concentration spatiale par secteurs d'activité. Les districts industriels italiens sont ainsi très vite devenus le modèle à suivre. Les politiques de clusters sont de plus apparues comme un moyen d'allier deux logiques de politiques industrielles présentées jusque là comme exclusives : une approche sectorielle, ciblant des industries précises, et une approche plus horizontale, ne cherchant pas à soutenir un secteur particulier mais à agir sur l'environnement productif des entreprises (à travers le rapprochement entre entreprises et laboratoires de recherche notamment). On assiste ainsi depuis le début des années 1990 à une multiplication de par le monde des politiques publiques visant à promouvoir les dynamiques de clusters : le Pays Basque espagnol, 
la Bavière, le Brésil, le Japon en sont quelques exemples. La France a de son côté développé ses politiques de clusters en deux temps, avec la mise en place des Systèmes Productifs Locaux (ci-après, 'SPL') tout d'abord en 1998, puis de la politique des Pôles de compétitivité, de plus grande envergure, en 2005. Dans cet engouement général pour les clusters comme outil de compétitivité et de développement régional, la Wallonie n'est pas en reste. Après avoir commencé à soutenir une douzaine de clusters dans les années 2000, la Wallonie se dote elle aussi d'une politique de pôles de compétitivité, axée sur les projets collaboratifs innovants, avec le Plan Marshall et le Plan Marshall2.vert (voir, à ce propos, le numéro 90 de Regards économiques).

Il devient toutefois difficile de s'orienter dans la galaxie des politiques de clusters. Conduites au niveau local, régional, national, présentées comme instruments de développement des régions à la traîne (et défendues à ce titre par la DG Regio au sein de l'Union européenne) ou comme politiques destinées à renforcer la compétitivité des régions motrices (et soutenues en ce sens par la DG Entreprises et Industrie), certains finissent par douter de la cohérence de ces initiatives. Martin et Sunley (2003) vont jusqu'à qualifier le concept de clusters de «chaotique». Une chose est certaine, les politiques de clusters mises en place un peu partout sont diverses. L'objectif visé est en effet toujours d'accroître, à travers le développement de projets collaboratifs, les performances des entreprises en termes d'innovation, de productivité ou encore d'exportations. Pour les pouvoirs publics les mettant en place, ces politiques doivent aussi avoir des retombées, à un niveau plus macroéconomique, sur l'emploi et la richesse nationale. Toutefois, les moyens mis en œuvre, les modalités d'attribution des aides et le public visé varient fortement d'une expérience à une autre.

L'évaluation économique des politiques de clusters est un moyen de mettre de l'ordre dans ce foisonnement. Grâce à l'utilisation de méthodes quantitatives adéquates, elle doit répondre à deux questions: :

1) Qui a été réellement aidé ? Il s'agit ici d'évaluer les caractéristiques des entités soutenues par la politique avant même qu'elles ne reçoivent une aide.

2) Quel a été l'impact de la politique sur les performances des entités aidées ? Il s'agit alors de comparer l'évolution d'un certain nombre d'indicateurs de performance à ce qu'ils auraient été en l'absence de politique publique.

Nous montrons dans ce numéro de Regards économiques qu'un discours généralisant sur les politiques de clusters est impossible, et que leur évaluation est affaire de cas par cas, en nous appuyant plus particulièrement sur les travaux que nous avons réalisés sur les SPL et les pôles de compétitivité français.

Après avoir indiqué pourquoi un décalage important peut exister entre le discours accompagnant la mise en place d'une politique industrielle et ce qui est réalisé, nous illustrons notre propos par l'analyse du ciblage de deux politiques de clusters françaises, les SPL et les pôles de compétitivité. Nous étudions ensuite l'impact des politiques de clusters sur les performances des entreprises. Si le soutien apporté aux clusters de biotechnologie en Allemagne semble avoir été bénéfique, nous montrons que la politique française des SPL n'a pas eu d'effet significatif sur la productivité, l'emploi ou les exportations des entreprises aidées. Des différences dans l'ampleur des moyens mobilisés et la nature des aides apportées peuvent expliquer ces résultats contrastés. 


\section{Politique industrielle et ciblage : du discours aux actes}

La mise en place d'une politique de clusters, comme de toute politique publique, s'accompagne d'un discours sur le public visé par le dispositif. La première question à laquelle doit alors s'intéresser l'évaluateur est celle de l'identification des entreprises effectivement aidées par les pouvoirs publics. Cette étape de l'analyse n'a rien de trivial, car un décalage est parfois observé entre les objectifs affichés en matière de ciblage et ce qu'une analyse statistique rigoureuse révèle avoir été réalisé.

Les études empiriques sur les politiques industrielles font souvent le constat que les bénéficiaires des aides publiques sont, même si telle n'était pas l'ambition de départ, des entreprises en difficulté. Alors que le Japon est par exemple régulièrement présenté comme un modèle en matière de politique industrielle ciblant les secteurs à fort potentiel de croissance, Beason et Weinstein (1996) mettent en lumière une réalité assez différente : au cours de la seconde moitié du XXème siècle, les secteurs à faible croissance et à rendements d'échelle décroissants ${ }^{1}$ (les mines et le secteur textile notamment) ont bénéficié au Japon d'aides publiques plus importantes que les autres secteurs. Plusieurs familles d'explications peuvent rendre compte de cette distorsion des aides en faveur des entreprises ou des secteurs les plus faibles.

Un premier ensemble d'arguments repose sur l'existence d'un biais dans les préférences des décideurs publics. Pour Corden (1974) par exemple, les pertes de revenus auraient un poids plus important que les gains dans les préférences des décideurs publics. Les gouvernements chercheraient ainsi à préserver les secteurs en perte de vitesse d'une totale dislocation, et non à favoriser le transfert des ressources productives de ces derniers vers les nouveaux secteurs à forte croissance. Krueger (1990) s'intéresse de son côté au fait que dans de nombreux pays, dont les Etats-Unis, les politiques commerciales favorisent généralement les secteurs souffrant de la concurrence internationale plutôt que les secteurs exportateurs disposant d'un avantage comparatif. Elle propose une explication aux différences de poids dans les préférences collectives invoquées par Corden (1974) basée sur le «biais d'identité»: on se préoccupe plus des personnes que l'on connaît que de celles que l'on ne connaît pas. Or, il est plus facile d'identifier les secteurs en difficulté, et donc les travailleurs dont l'emploi est menacé, que les secteurs en forte progression. Dès lors, les politiques industrielles souffriraient elles-mêmes d'un biais systématique en faveur des secteurs à la traîne.

Ces explications postulent directement l'existence du biais observé statistiquement dans plusieurs pays plus qu'elles ne rendent compte de la formation de ce biais. Des explications récentes mettent en avant des mécanismes plus endogènes. Baldwin et Robert-Nicoud (2007) enrichissent par exemple le modèle de lobbying de Grossman et Helpman (1994). Les décideurs politiques sont influencés par les groupes de pression qui dépensent de l'argent pour promouvoir leurs intérêts. Toutefois, dans des secteurs en expansion, le retour sur investissement que les lobbyistes peuvent attendre de leurs dépenses est assez faible. En effet, quand bien même les pouvoirs publics prennent des mesures favorables au secteur d'activité des lobbyistes, la rente ainsi créée doit être partagée entre des bénéficiaires plus nombreux, du fait du nombre croissant d'entreprises actives dans le secteur. $\mathrm{Au}$ contraire, dans les industries en déclin et avec des barrières à l'entrée suffisamment élevées, la rente obtenue grâce aux activités de lobbying est plus facilement

\footnotetext{
${ }^{1}$ La production dans un secteur se fait à rendements d'échelle croissants lorsque la production augmente de manière plus que proportionnelle suite à un accroissement des quantités de facteurs de production utilisées. D'après les calculs de Beason et Weinstein (1996), les industries extractives et textiles, fortement soutenues par les pouvoirs publics japonais, présentent au contraire des rendements d'échelle décroissants, si bien que la stimulation de la production dans ces secteurs ne sera pas génératrice de gains d'efficacité (à technologie constante).
} 


\author{
... Politique industrielle et ciblage : \\ du discours aux actes
}

appropriable par les entreprises déjà en place. Au final, ce sont donc les secteurs en perte de vitesse qui ont le plus intérêt à se livrer aux activités de lobbying et qui bénéficient de manière privilégiée d'interventions publiques visant à les protéger.

Baldwin et Okubo (2006) s'intéressent plus particulièrement aux subventions allouées aux entreprises pour venir se localiser dans des régions dont les pouvoirs publics veulent renforcer l'attractivité (Prime d'Aménagement du Territoire en France, Regional Selective Assistance en Angleterre, Zones Franches en Wallonie). Ces régions sont la plupart du temps des zones peu denses souffrant d'un déficit d'image. Baldwin et Okubo (2006) montrent que ces subventions à la localisation peuvent avoir comme conséquence inattendue d'attirer vers les régions aidées les entreprises les moins productives. En effet, il est bien connu en économie géographique et urbaine que les entreprises gagnent à être localisées dans des zones denses, proches des marchés qui constituent leurs débouchés, mais aussi d'autres entreprises afin de bénéficier d'externalités positives en termes de production. Dès lors, une entreprise n'acceptera de quitter une zone dense pour la périphérie que si le montant de la subvention compense au moins les pertes en termes d'accès aux marchés et d'externalités que ce départ occasionne. Si les entreprises les plus productives sont celles qui gagnent le plus à être localisées dans les zones denses, les subventions à la localisation risquent de n'attirer vers les régions à la traîne que les entreprises les moins performantes, et ce d'autant plus que le montant de la subvention est faible. La nature même des incitations mises en œuvre peut ainsi induire une forme d'auto-sélection des entreprises dans le dispositif proposé. Les résultats que mesurent les économistes en matière de ciblage ne révèlent donc pas nécessairement des préférences particulières des pouvoirs publics, mais peuvent aussi être le fruit endogène et inattendu des incitations mises en place.

L'extrême diversité des politiques de clusters, tant du point de vue des institutions qui les mettent en place (Etats, régions, communes) que de la nature des aides proposées (subventions de structures d'animation collective, financement d'infrastructures, aides à la R\&D collaborative etc.), laisse présager d'une grande hétérogénéité dans les publics touchés par ces dispositifs. C'est ce que révèle l'analyse de deux politiques de clusters conduites en France, la politique des Systèmes Productifs Locaux et la politique des Pôles de compétitivité.

La politique des SPL a été mise en place en France à la fin des années 1990 par la Délégation à l'Aménagement du Territoire et à l'Action Régionale (DATAR), administration en charge de la politique régionale. Elle devait marquer un changement profond dans la logique sous-tendant les politiques régionales en France. En effet, alors que les pouvoirs publics français cherchaient jusqu'alors, dans un objectif d'équité, à re-disperser les activités qu'ils jugeaient trop concentrées autour de Paris, la politique des SPL prenait acte des gains liés à l'agglomération spatiale de la production, et visait au contraire à favoriser les dynamiques de clusters. Plus précisément, une subvention relativement modeste (autour de 40.000 euros en moyenne) était versée à une structure collective (association, groupement d'intérêt économique ou autre) portant une action commune à plusieurs entreprises. Les actions ainsi soutenues pouvaient être très différentes, de la création d'un label collectif à la mise en place d'un groupement d'employeurs par exemple. Dans l'esprit des pouvoirs publics de l'époque, la subvention monétaire n'était qu'une incitation visant à enclencher des collaborations durables entre entreprises d'un même secteur qui, bien que proches géographiquement, ne communiquaient pas jusqu'alors. La sélection s'est opérée par appel à projets. La DATAR a ainsi choisi à la fin de 1998 une cinquantaine de projets sur une centaine reçus. L'expérience a 


\section{... Les Systèmes Productifs Locaux}

été renouvelée à la fin de l'année $1999 .^{2}$ Les dossiers étaient déposés par la structure collective porteuse de l'action.

Martin et al. (2011) réalisent une évaluation de cette politique. Avant d'en étudier l'impact, ils identifient qui a réellement été aidé : ils déterminent les caractéristiques des entreprises soutenues avant même que ces dernières ne bénéficient de la subvention. Pour ce faire, il a été demandé aux différents SPL de transmettre la liste de leurs membres. ${ }^{3}$ Cette dernière a été appariée aux données administratives de bilan des entreprises industrielles de plus de 20 salariés transmises par le ministère de l'Industrie (cf. encadré 1 pour plus de détails sur la méthodologie utilisée).

L'analyse montre que les entreprises des SPL sont plus grosses que les autres en termes de chiffre d'affaires, mais aussi, à taille donnée, moins productives en termes de productivité totale des facteurs. Toutefois, ce différentiel négatif de productivité est entièrement dû au fait que les entreprises des SPL appartiennent à des secteurs et des territoires en perte de vitesse. Dès lors que leur secteur d'activité et leur localisation sont pris en considération, les entreprises des SPL demeurent plus grosses que les autres, mais ne sont pas statistiquement différentes en termes de productivité totale des facteurs. Il apparaît aussi que les entreprises des SPL perçoivent, avant même leur entrée dans le dispositif, plus de subventions que les autres. Ce sont donc des entreprises déjà aidées par ailleurs. Enfin, les auteurs montrent que si les entreprises des SPL appartiennent bien à des clusters, il s'agit de clusters d'envergure locale et pas nationale.

L'étude du public effectivement ciblé par la politique des SPL révèle donc que cette dernière n'a pas marqué de réelle rupture dans les politiques d'aménagement du territoire en France. Elle marque au contraire une certaine forme de continuité. Les pouvoirs publics ont soutenu les secteurs et les territoires les plus en difficulté, et promu les dynamiques de clusters au sein d'agglomérations productives importantes au niveau local, mais pas au niveau national. L'analyse des effets de sélection à l'œuvre avec la politique des SPL illustre donc bien le décalage qui peut exister entre les objectifs affichés et les objectifs révélés d'une politique.

Difficile toutefois de savoir si ces résultats sont le reflet de préférences particulières des décideurs publics ou d'une auto-sélection des entreprises. En effet, la labellisation des SPL s'est opérée par appels à projets, mode de mise en œuvre des politiques industrielles de plus en plus courant. Si les pouvoirs publics ont bien effectué le choix final, ils n'ont pu le faire que parmi les répondants. La forme même de l'appel à projets conduit donc à un double niveau de sélection que l'évaluateur ne peut pas toujours dissocier : la sélection opérée par l'organisation en charge de la politique est précédée d'une auto-sélection des répondants.

\section{- Identification des caractéristiques des entreprises aidées et choix du groupe de référence}

D'un point de vue statistique, pour déterminer les caractéristiques qui influent sur la probabilité qu'une entreprise appartienne à un SPL, on utilise des modèles non linéaires de type logit ou probit. Lors de cette étape, le choix du groupe de référence s'avère absolument fondamental. En effet, lorsqu'on compare de manière brute les entreprises SPL à toutes les autres entreprises industrielles qui ne sont pas membres d'un SPL, on s'aperçoit que les entreprises des SPL sont plus grosses en termes de chiffre d'affaires, mais

\footnotetext{
${ }^{2}$ Transformée en appel à projets permanent, cette politique a été abandonnée au milieu des années 2000 et reprise en 2009 sous une forme un peu différente.

${ }^{3}$ Cf. Martin et al. (2011) pour plus de détails sur la construction de la base de données.
} 


\section{- ... Identification des caractéristiques des entreprises aidées et choix du groupe de référence}

2.2. Les pôles de compétitivité à taille donnée (en termes de chiffre d'affaire), moins productives (en termes de productivité totale des facteurs). Toutefois, dès lors que l'on compare les entreprises SPL aux entreprises du même secteur d'activité, le différentiel de productivité n'est plus statistiquement significatif : on ne peut pas rejeter I'hypothèse que la productivité des entreprises SPL soit la même que celle des entreprises hors SPL à un seuil de confiance de $10 \%$. Lorsque I'on tient compte en plus du département où elles sont localisées, ce différentiel devient même légèrement positif, quoique non statistiquement significatif. Le différentiel de taille positif entre les entreprises SPL et les autres ne fait au contraire que s'accentuer au fur et à mesure que l'on affine le groupe de comparaison. La comparaison des résultats obtenus suivant le groupe de contrôle retenu indique donc que les entreprises des SPL appartiennent à des secteurs et se trouvent dans des départements où les entreprises sont plus petites et moins productives que l'entreprise française «moyenne» (puisque le coefficient obtenu sur le chiffre d'affaires et la productivité totale des facteurs augmente lorsqu'on affine le groupe de contrôle d'un point de vue géographique et sectoriel). En revanche, quel que soit le référentiel utilisé, les résultats de Martin et al. (2011) montrent que les entreprises des SPL sont des entreprises qui, avant même de bénéficier de la politique, touchent plus de subventions publiques que les autres.

Par ailleurs, la politique des SPL étant une politique de clusters, les auteurs regardent si le fait d'avoir un nombre élevé d'entreprises de son propre secteur situées dans le même département accroît la probabilité qu'une entreprise fasse partie d'un SPL financé par I'Etat. Paradoxalement, lorsqu'on prend comme groupe de comparaison l'ensemble des entreprises industrielles ou les entreprises du même secteur uniquement, c'est le contraire que l'on observe. Ce n'est que lorsque I'on tient compte du département où elles sont situées que le nombre d'entreprises du même secteur situées à proximité accroît significativement la probabilité qu'une entreprise fasse partie d'un SPL. L'interprétation de ce résultat est simple : les SPL sont des clusters d'envergure locale, et non nationale. Ils représentent une masse critique d'entreprises au sein des départements où ils sont situés, mais pas à l'échelle du pays ou du secteur.
Il est néanmoins possible d'aller plus loin dans la distinction de ces deux niveaux de sélection avec les pôles de compétitivité français. En effet, la sélection des pôles de compétitivité à l'été 2005 a elle aussi été réalisée à l'issue d'un appel à projets. La politique des pôles est toutefois très différente de celle des SPL. La nature des aides publiques proposées n'est tout d'abord pas la même, l'essentiel des crédits alloués consistant en des subventions à des projets collaboratifs de $R \& D$ impliquant au moins deux entreprises et un laboratoire de recherche. Le design de la politique en deux étapes permet de dissocier plus clairement sélection et auto-sélection. Un comité interministériel a dans un premier temps, sur la base des dossiers de candidature reçus, labellisé les pôles, c'est-à-dire choisi des couples secteur-territoire au sein desquels les entreprises seront éligibles aux subventions accordées au titre de la politique des pôles : l'aéronautique à Toulouse, la microélectronique dans la région grenobloise, l'industrie des logiciels en Ile-deFrance etc. Plus d'une soixantaine de dossiers ont été retenus sur une centaine qui 


\section{... Les pôles de compétitivité}

avaient été déposés. Afin de marquer néanmoins une hiérarchie dans la sélection, trois catégories de pôles ont été distinguées par les pouvoirs publics, par ordre décroissant d'ambition : les pôles mondiaux, les pôles à vocation mondiale et les pôles nationaux. Ce n'est que dans un deuxième temps que les entreprises se sont adressées aux structures de gouvernance des pôles pour en devenir membres. Seuls les membres des pôles peuvent en effet candidater aux appels à projets R\&D lancés après la labellisation pour distribuer les subventions. ${ }^{4}$ Deux questions peuvent alors être posées par l'évaluateur : au sein d'un secteur donné, est-ce que les pouvoirs publics français ont choisi les territoires les plus performants pour devenir pôles de compétitivité ? Et au sein d'un couple secteur-territoire bénéficiant de la politique des pôles, est-ce que ce sont les entreprises les plus performantes qui se sont auto-sélectionnées pour devenir membres des pôles ?

C'est la démarche que suivent Fontagné et al. (2010), qui s'intéressent plus particulièrement aux performances à l'exportation des entreprises des pôles de compétitivité en 2004, l'année précédant le lancement de la politique. Pour ce faire, ils apparient la liste des entreprises membres des pôles de compétitivité établie par le ministère de l'Industrie en 2006 avec les données de bilan et les données d'exportation des entreprises françaises. Il apparaît très clairement que les entreprises localisées dans les secteur-département bénéficiant d'un pôle de compétitivité sont plus productives et exportent plus de produits, vers plus de pays, plus loin, en plus grande quantité, et à un prix plus élevé que les entreprises du même secteur localisées dans les autres départements. La hiérarchie établie lors de la labellisation entre les trois catégories de pôles de compétitivité se trouve par ailleurs confirmée par l'analyse statistique. La première conclusion de cette étude est donc qu'au sein d'un secteur donné, l'appel à projets a permis aux pouvoirs publics de choisir les meilleurs endroits pour y établir les pôles de compétitivité.

Par ailleurs, au sein des secteurs et territoires ainsi définis, il apparaît que ce sont aussi les entreprises ayant les meilleures performances en termes de productivité et d'exportation qui se sont auto-sélectionnées pour devenir membres des pôles de compétitivité. Là encore, la hiérarchie établie par les pouvoirs publics est vérifiée, les entreprises des pôles mondiaux ayant de meilleures performances que celles des deux autres catégories de pôles. Toutefois, il semble qu'au sein des pôles mondiaux, cette «surperformance» à l'exportation soit presqu'entièrement due au fait que les entreprises en question sont plus grosses (en termes d'employés) et plus productives (en termes de productivité totale des facteurs) que les autres. Cette catégorie de pôle regroupe donc les fleurons de l'industrie française, qui étaient en réalité identifiables bien avant la mise en place de la politique. En revanche, les entreprises des pôles nationaux continuent de présenter, à taille et productivité données, de meilleures performances à l'exportation que les autres entreprises du même secteur et du même département. Cette catégorie de pôles semble ainsi regrouper des entreprises jouissant d'un «génie» particulier (produit de niche, qualité supérieure, stratégie commerciale offensive etc.) leur permettant d'exporter au-delà de ce que leur taille et leur productivité prédisent. Les résultats sont exactement les mêmes si l'on considère l'évolution des performances des entreprises entre 2001 et 2004 et pas seulement leur niveau en 2004. La deuxième conclusion de cette étude est ainsi qu'au sein des territoires et secteurs bénéficiant du label pôle de compétitivité, ce sont les meilleures entreprises qui se sont autosélectionnées au sein des pôles, tant d'un point de vue statique que dynamique. Le diagnostic posé sur la politique des pôles est donc bien différent de celui posé pour les SPL. Nous proposons maintenant quelques pistes d'explications possibles.

\footnotetext{
${ }^{4}$ La première phase de la politique couvrait la période 2005-2008 et était dotée d'un budget total de 1,5 milliards d'euros. Suite à une évaluation réalisée par les cabinets de conseil BCG et CM International, une deuxième phase de trois ans a été dotée du même budget. Une nouvelle évaluation doit avoir lieu en 2012.
} 


\section{Politiques de clusters, incitations et sélection}

Il est difficile de se prononcer en termes normatifs sur le type de ciblage que les politiques de clusters devraient adopter. Toute politique régionale fait face à un dilemme équité/efficacité : faut-il aider les territoires ou les entreprises à la traîne, même si nombre de ces derniers ne parviendront sans doute pas à se hisser aux avant-postes de leur domaine, ou au contraire concentrer les moyens sur ceux qui font d'ores et déjà état de bonnes performances, afin d'assurer et renforcer leur position? Idéalement, les politiques publiques devraient sans doute identifier les activités et entreprises prometteuses qui en raison de barrières à l'entrée, de contraintes de crédit ou autre frein à leur développement, nécessitent une intervention publique pour donner leur pleine mesure. Au cas présent, l'analyse des SPL et des pôles de compétitivité français révèle de forts contrastes entre les deux politiques. La première s'apparente à une politique régionale traditionnelle venant soutenir des entreprises opérant dans des secteurs et des territoires en difficulté tandis que la seconde cible au contraire des territoires et des entreprises moteurs au sein d'un secteur donné.

L'étude des SPL et des pôles de compétitivité montre ainsi que des politiques très différentes sont regroupée sous le label de «politiques de clusters». Elle révèle aussi que la volonté des pouvoirs publics, mais aussi les modalités de mise en œuvre des politiques proposées, déterminent simultanément, et parfois dans des sens opposés, les bénéficiaires des politiques de clusters, et plus généralement des politiques industrielles. La politique des SPL était conduite par la DATAR, administration en charge des politiques régionales en France, tandis que la politique des pôles de compétitivité est menée conjointement par la DATAR et le ministère de l'Industrie, ce dernier ayant une approche plus liée à la filière qu'au territoire.. Ces différences de tutelle dans la gestion des deux politiques expliquent sans doute partiellement le contraste dans leurs bénéficiaires. Par ailleurs, les incitations proposées étaient fort différentes pour les SPL et pour les pôles. Pour les SPL, les sommes en jeu étaient relativement modestes et couvraient un spectre d'actions éligibles très large, parfois peu opérationnelles. La majeure partie des aides allouées au titre de la politique des pôles de compétitivité prennent au contraire la forme de subventions à la $R \& D$ conséquentes attribuées par appels à projets spécifiques (1,5 milliards d'euros ont été alloués à la politique de 2005 à 2008, puis à nouveau de 2009 à 2012). Seules les entreprises faisant de la R\&D ou à même de collaborer avec des laboratoires ou d'autres entreprises engagées dans des activités de $R \& D$ peuvent réellement bénéficier de ces aides. Or les entreprises faisant de la R\&D sont clairement plus performantes que les autres en termes de productivité et d'exportations (Aw et al., 2010). Dans la lignée des prédictions de Baldwin et Okubo (2006) sur les subventions à la localisation, il est donc probable que les incitations proposées par les SPL et les pôles de compétitivité aient conditionné pour partie le type d'entreprises qui ont souhaité participer à ces clusters différents.

Les pouvoirs publics attendent de la mise en œuvre des politiques de clusters un retour sur investissement à travers une amélioration des performances des entreprises aidées. Si les objectifs ne sont pas toujours clairement identifiés, les attentes concernent généralement l'innovation, l'emploi, les exportations et la productivité. La seconde question que doit donc se poser l'évaluateur est la suivante : quelles auraient été les performances des entreprises aidées si ces dernières n'avaient pas bénéficié de subventions ? Par définition, il n'est pas possible d'observer ce qu'auraient été les performances des entreprises aidées en l'absence de subventions. Le travail de l'évaluateur consiste donc de trouver une approximation raisonnable de ces dernières. Se pose à nouveau ainsi la question du groupe de comparaison.

\section{Impact des politiques de clusters sur les performances des entreprises aidées}




\subsection{Des évaluations économiques d'impact peu nombreuses}

La plupart des évaluations de politiques de clusters, réalisées pour les pouvoirs publics ou dans un cadre plus académique, sont des études qualitatives et descriptives. Elles s'intéressent aux aspects procéduraux de la mise en œuvre de ces politiques, à l'analyse de collaborations mises en place au sein de certains clusters, ou à la genèse et la conduite de projets particuliers. Très peu d'études en revanche tentent de mesurer l'impact moyen de ces politiques sur des variables de performances des entreprises ou des régions soutenues. A notre connaissance, deux évaluations de ce type ont été réalisées sur des politiques de clusters conduites en Allemagne.

Falck et al. (2010) s'intéressent à une politique de clusters conduite en Bavière à partir de 1999. Plusieurs secteurs, tels que les sciences de la vie, la mécatronique ou les technologies de l'innovation sont visés par cette politique d'investissement dans les infrastructures de recherche et de mise en réseau des acteurs publics et privés de l'innovation. Les auteurs concluent que la politique a accru la probabilité que les entreprises bavaroises touchées par la politique innovent de $5 \%$ environ.

Engel et al. (2011) évaluent deux politiques de clusters conduites au niveau fédéral en Allemagne pour le secteur des biotechnologies, BioRegio et BioProfile. Ils montrent que la politique a eu un impact positif et significatif sur le nombre de brevets déposés et sur le nombre de projets R\&D subventionnés conduits dans les régions concernées par le dispositif.

Si les expériences allemandes semblent avoir été positives pour la dynamique d'innovation, les résultats des SPL sur les performances des entreprises sont moins encourageants.
Il n'existe pas encore d'étude quantitative cherchant à mesurer l'impact des pôles de compétitivité français, le recul temporel par rapport à la mise en place de la politique étant jusqu'à aujourd'hui insuffisant. Ce travail a en revanche été effectué pour les SPL par Martin et al. (2011). Ainsi qu'en attestent les graphiques de la figure 1 (voir page 11), les entreprises aidées sont en réalité sur une pente décroissante en termes de productivité. Bien que peu différentes des entreprises non-aidées au départ, elles deviennent statistiquement moins productives au cours de la période. Il ne faut toutefois pas voir dans ces performances décevantes un impact négatif de la politique des SPL. Ces dernières sont largement imputables au secteur d'activité et à la zone géographique où sont localisés les SPL. Il semble par ailleurs que les entreprises entrant dans le dispositif soient des entreprises faisant face à des difficultés particulières. Dès lors que l'on tient compte de ces biais de sélection et de simultanéité, la politique des SPL ne semble pas avoir eu d'impact, ni sur la productivité, ni sur l'emploi ou les exportations des entreprises aidées (cf. encadré 2 pour les aspects méthodologiques).

Les résultats obtenus sur les SPL français ne peuvent certes pas être extrapolés aux pôles de compétitivité ou à d'autres politiques de cluster conduites ailleurs dans le monde. Ils invitent néanmoins à la prudence vis-à-vis de politiques qui sont souvent présentées aujourd'hui comme un remède miracle aux problèmes de compétitivité ou d'attractivité dont souffrent certaines régions ou pays.

La faiblesse des moyens mis en œuvre explique sans doute en partie l'absence d'impact significatif des SPL. Le coût total des deux premières vagues de labellisation des SPL était inférieur à 5.000.000 euros (40.000 euros de subvention en moyenne par SPL pour une centaine de SPL soutenus). En comparaison, la stratégie de clusters conduite en Bavière a bénéficié de 1,35 milliard d'euros, tandis que les politiques BioRegio et BioProfile disposaient respectivement d'un budget de 750 et 150 millions d'euros. Nous ne suggérons pas ici que de plus grosses dépenses sont nécessairement le gage d'une politique réussie, mais qu'il existe sans doute un seuil en dessous duquel une subvention monétaire peine à jouer son rôle d'incitant. Ainsi que nous l'avons déjà évoqué, des subventions faibles peuvent 
aussi conduire à une autosélection des entreprises les moins performantes dans le dispositif. Or le ciblage par les SPL d'entreprises rencontrant des difficultés importantes explique sans doute en partie les résultats obtenus.

\section{․ Mesure d'impact \\ 崖 et traitement des biais de sélection et de simultanéité}

Les graphiques de la figure 1 illustrent parfaitement les enjeux liés à la définition du groupe de contrôle adéquat pour l'étude d'impact. Ces graphiques montrent l'évolution du différentiel de productivité entre les entreprises des SPL et les autres, avant et après I'obtention de la subvention. Le premier graphique montre que si les entreprises des SPL semblent un peu moins productives avant même qu'elles ne jouissent de la politique, cet écart négatif n'est pas significativement différent de zéro d'un point de vue statistique (la bande grise donnant l'intervalle de confiance statistique de cet écart, c'est-à-dire la différence entre la valeur la plus faible et la valeur la plus élevée possible de cet écart). II devient statistiquement significatif à partir de l'année d'entrée dans les SPL et va croissant au cours des cinq années qui suivent. Est-ce à dire que la politique des SPL a un impact négatif sur la productivité des entreprises qui en sont membres ? Le second graphique présente la même évolution une fois que l'on a pris en compte le secteur d'activité et le département où l'entreprise est localisée. Cela revient en quelque sorte à réduire le groupe de comparaison aux entreprises non SPL des secteurs et départements concernés par la politique. Le déclin devient alors bien moins marqué et l'écart de productivité avec les autres entreprises n'est plus statistiquement significatif. On retrouve ainsi l'idée que les entreprises SPL appartiennent à des secteurs et des départements en perte de vitesse. Au final, la méthode économétrique de la «différence de différences», consistant à comparer l'évolution de la productivité des entreprises aidées à cette même évolution pour les entreprises non-aidées, laisse penser à un impact très légèrement négatif de la politique des SPL. Ce coefficient négatif, un peu surprenant compte tenu du caractère peu contraignant de la politique, peut être imputable à un déclin des entreprises SPL amorcé avant ou au moment de la mise en place du dispositif, ce que semble confirmer l'analyse graphique précédente. II y aurait alors un biais de simultanéité.

Plusieurs solutions s'offrent à l'évaluateur pour tenter de remédier au biais de simultanéité. En particulier, la méthode de matching consiste à réduire le groupe de contrôle à des entreprises non subventionnées disposant néanmoins d'une probabilité ex ante élevée de bénéficier du dispositif. Si cette probabilité est bien corrélée aux chocs inobservables touchant les entreprises peu avant ou en même temps que la mise en œuvre de la politique, la méthode de matching peut aider à corriger le biais de simultanéité. Ce calcul de probabilité s'effectue sur la base du travail effectué en première étape concernant la caractérisation des entreprises aidées. D'autres méthodes sont aussi envisageables, telles que la prise en compte d'effets conjoncturels spécifiques aux secteurs ou la modélisation d'une corrélation dans les performances des entreprises au cours du temps.

Quelle que soit la méthode utilisée, après avoir corrigé ce biais, Martin et al. (2011) ne trouvent aucun impact de la politique des SPL sur la productivité totale des facteurs des entreprises. Ils ne détectent pas d'effet significatif non plus sur l'emploi ou les exportations. 
Figure 1. Différentiel de productivité entreprises SPL et non SPL

Source : Duranton et al. (2008).
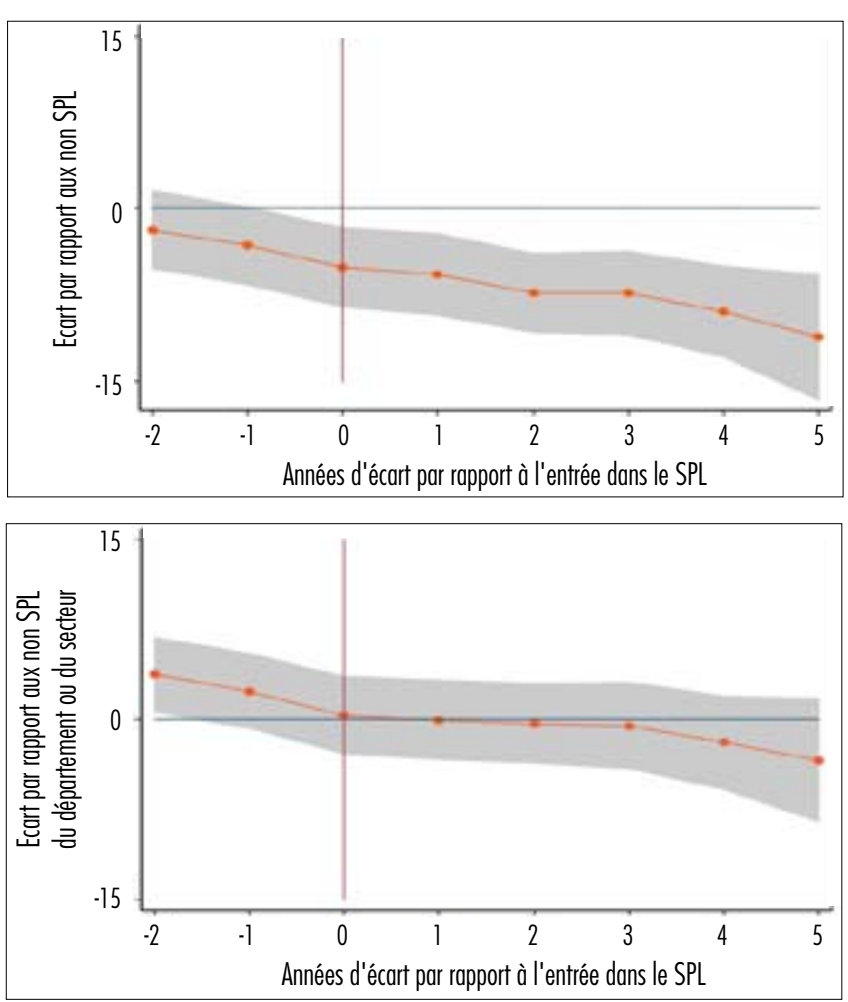

Les politiques de clusters sont donc très diverses dans les publics qu'elles visent et les modalités de leur mise en œuvre. Il est de ce fait impossible aujourd'hui de tenir un discours univoque sur leur bien-fondé ou leur efficacité. En ce sens, l'évaluation des politiques de clusters est nécessaire pour mettre de l'ordre dans la diversité des expériences conduites. D'un point de vue méthodologique, deux étapes sont nécessaires à une évaluation économique des politiques clusters : l'identification des caractéristiques particulières des entreprises soutenues et la mesure de l'impact. Loin d'être indépendantes, ces deux étapes sont au contraire intimement liées. L'analyse du processus de sélection permet de nourrir la réflexion sur le groupe de contrôle indispensable à la mesure de l'impact, mais aussi de repositionner la politique évaluée par rapport à ses objectifs initiaux. Il peut en effet exister des décalages entre les ambitions de départ d'une politique et ce qui est réalisé. Ces décalages peuvent naître d'une volonté délibérée des pouvoirs publics ou être la conséquence inattendue des incitations mises en place. En ce sens, l'évaluation des politiques de clusters à partir de méthodes quantitatives ne vient pas seulement mesurer et sanctionner un résultat. Elle fournit aussi un éclairage sur le dispositif même de la politique, utile à son évolution et à son amélioration. Un dernier aspect n'a pas été évoqué, la mesure du coût et des bénéfices de ces politiques, mettant en rapport les moyens mis en œuvre et l'impact obtenu. Une évaluation économique devrait en effet permettre de quantifier combien d'euros ont été générés par euro dépensé. Le caractère multidimensionnel des politiques de cluster (innovation, productivité, emploi, exportations etc.) rend difficile cet exercice et explique sans doute que nous ne disposions pas de telles mesures à ce jour. Il s'agit là d'un défi à relever pour les évaluations à venir. 


\section{Références}

Directeur de la publication :

Vincent Bodart

Rédactrice en chef :

Muriel Dejemeppe

Comité de rédaction : Paul Belleflamme,

Vincent Bodart, Thierry Bréchet,

Muriel Dejemeppe, Frédéric Docquier,

Jean Hindriks, Marthe Nyssens

Secrétariat \& logistique : Anne Davister

Graphiste : Dominos
Aw, B. Y., M. J. Roberts, et D. Y. Xu. 2010. R\&D Investment, Exporting, and Productivity Dynamics. A paraître dans American Economic Review.

Baldwin R.E. et T. Okubo. 2006. Heterogeneous firms, agglomeration and economic geography: spatial selection and sorting. Journal of Economic Geography, 6(3), 323-346, June 2006

Baldwin, R. E., et F. Robert-Nicoud. 2007. Entry and Asymmetric Lobbying: Why Governments Pick Losers. Journal of the European Economic Association, 5(5), 10641093.

Beason, R. et D. E. Weinstein. 1996. Growth, economies of scale, and targeting in Japan (1955-1990). The Review of Economics and Statistics, 78(2), 286-295.

Corden, M. 1974. Trade Policy and economic Welfare. Oxford University Press, Oxford.

Duranton, G., P. Martin, T. Mayer et F. Mayneris. 2008. Les pôles de compétitivité. Que peut-on en attendre? Collection du Cepremap, Editions Rue d'Ulm

Engel, D., T. Mitze, R. Patuelli et J. Reinkowski. 2011. Does the support of innovative clusters sustainably foster R\&D activity? Evidence from the German BioRegio and BioProfile contests. Rimini Centre for Economic Analysis WP 11-15.

Grossman, G. et E. Helpman. 1994. Protection for Sale. American Economic Review, 84(4), 833-850.

Falck, O., S. Kipar et S. Heblich. 2010. Industrial innovation: Direct evidence from a cluster-oriented policy. Regional Science and Urban Economics, 40(6), 574-582.

Fontagné, L., P. Koenig, F. Mayneris et S. Poncet. 2010. Clustering the Winners: An Assessment of the French Competitiveness Clusters Policy. CEPII DP 2010-18.

Krueger, A. 1990. Asymmetries in policy between exportable and import-competing goods, in The political economy of international trade, ed. by R. Jones, and A. Krueger. Basil Blackwell.

Marshall, Alfred. 1890. Principles of Economics. London: Macmillan.

Martin, P., Mayer, T., et Mayneris, F., 2011. Public support to clusters: A firm-level study of French Local Productive Systems, 1996-2004. Regional Science and Urban Economics, 41(2), 108-123.

Martin, R. et P. Sunley. 2003. Deconstructing clusters: Chaotic concept or policy panacea. Journal of Economic Geography, 3(1), 5-35.

Porter, M. 2000. Location, competition, and economic development: Local clusters in a global Economy. Economic Development Quarterly, 14(1),15-34.
Regards Économiques IRES-UCL

Place Montesquieu, 3

B1348 Louvain-la-Neuve

http://www.uclouvain.be/en-regards-economiques

regard-ires@uclouvain.be

tél. $010 / 473426$
ISSN 2033-3013 